Open Access

\title{
Effects of exercise training and photobiomodulation therapy (EXTRAPHOTO) on pain in women with fibromyalgia and temporomandibular disorder: study protocol for a randomized controlled trial
}

Mariana Moreira da Silva*, Regiane Albertini, Ernesto Cesar Pinto Leal-Junior, Paulo de Tarso Camillo de Carvalho, José Antonio Silva Jr, Sandra Kalil Bussadori, Luis Vicente Franco de Oliveira, Cezar Augusto Souza Casarin, Erinaldo Luiz Andrade, Danilo Sales Bocalini and Andrey Jorge Serra

\begin{abstract}
Background: Fibromyalgia (FM) is a syndrome most prevalent in women, in whom it is characterized mainly by chronic pain. An important issue is that many patients with FM are reported to have temporomandibular dysfunction (TMD), and the coexistence of these pathologies generates a clinical outcome of high complexity. The literature is unclear regarding an effective therapy for reducing pain in patients with both comorbidities. Exercise training and phototherapy (low-level laser therapy with light-emitting diode) are two of the approaches used to treat pain. Thus, the aim of this study is to assess the potential role of exercise training plus phototherapy in reducing chronic pain in women with FM and TMD. A further aim is to determine whether the interventions can improve quality of life and modulate endogenous serotonin.

Methods/Design: A randomized controlled clinical trial will be conducted. It will involve 60 women $\geq 35$ years of age with a diagnosis of FM and TMD. After recruitment, patients will be randomly allocated to one of four groups: a control group (no intervention), a group that will receive a phototherapy intervention ( $\mathrm{PHO}$ ), a group that will be prescribed muscle-stretching, aerobic, and facial exercises (EXT), or a group that will receive phototherapy plus exercise interventions $(\mathrm{PHO}+\mathrm{EXT})$. The trial will last 10 weeks, and the following outcomes will be evaluated on two separate occasions (baseline and within $24 \mathrm{~h}$ after the last day of the protocol). Pain intensity will be analyzed using a visual analogue scale and the McGill Pain Questionnaire, and pain thresholds will be punctuated using a digital algometer. FM symptoms will be assessed using the Fibromyalgia Impact Questionnaire, and quality of life will be determined with the 36-item Short Form Health Survey. Serotonin levels will be evaluated in salivary samples using a competitive enzyme-linked immunosorbent assay.
\end{abstract}

Discussion: This is the first randomized controlled trial in which the role of phototherapy, exercise training, and a combination of these interventions will be evaluated for chronic pain in patients with FM and TMD. The results will offer valuable clinical evidence for objective assessment of the potential benefits and risks of procedures.

Trial registration: ClinicalTrials.gov Identifier: NCT02279225. Registered 27 October 2014.

Keywords: Exercise training, Fibromyalgia, Light-emitting diode, Low-level laser therapy, Pain, Phototherapy, Temporomandibular joint disorder

\footnotetext{
* Correspondence: fisioterapeutamariana@gmail.com

Nove de Julho University, Rua Vergueiro, 235, Liberdade, São Paulo, SP 01504-000, Brazil
}

\section{Biomed Central}

(c) 2015 da Silva et al. This is an Open Access article distributed under the terms of the Creative Commons Attribution License (http://creativecommons.org/licenses/by/4.0), which permits unrestricted use, distribution, and reproduction in any medium, provided the original work is properly credited. The Creative Commons Public Domain Dedication waiver (http:// creativecommons.org/publicdomain/zero/1.0/) applies to the data made available in this article, unless otherwise stated. 


\section{Background}

Fibromyalgia (FM) is a chronic syndrome characterized mainly by pain, nonrestorative sleep, fatigue, morning stiffness, depression, and cognitive disorders $[1,2]$. Patients with FM often show widespread pain in the presence of tender points (expressed as mild or greater tenderness), which provide the most sensitive, specific, and accurate criteria for making the diagnosis $[1,3]$. Using this classification, almost all patients with FM are women because they have more tender points than do men [4]. Overall, FM symptoms lead to significant reduction in functional capacity and quality of life $[2,5,6]$. Developing treatment teams is useful, including clinicians with expertise in patient education, exercise training interventions, and cognitive behavioral therapy [7-9]. An optimized treatment can be provided using the following steps: (1) overall recommendation (e.g., patient education), (2) nonpharmacologic (e.g., exercise, cognitive behavioral therapy, alternative medicine, and central nervous system neurostimulatory intervention), and (3) pharmacologic (e.g., tricyclic complexes, gabapentinoids, $\gamma$-hydroxybutyrate, naltrexone, cannabinoids, selective serotonin reuptake antagonists, nonsteroidal anti-inflammatory drugs, and opioids) [4].

A key issue is the association of FM with other musculoskeletal comorbidities, such as temporomandibular dysfunction (TMD) [5]. It has been shown that TMD can develop as a result of mandibular compression during daily living activities and sleep in the patients with FM [5-7], in which the coexistence of these pathologies generates a clinical outcome of high complexity [8]. Unfortunately, no published studies of patients with FM and TMD have evaluated an effective pharmacologic and/or nonpharmacologic therapeutic intervention. Previous studies have examined only the role of different interventions in the situation of a pathology per se. In this issue, exercise training is highly recommended in the controlling of patients with FM. Aerobic, strength, and mixed training programs (combination of aerobic, strength, and flexibility) were shown to reduce pain, number of tender points, fatigue, depression, and anxiety and to improve health-related quality of life as well as functional capacity $[9,10]$. Exercises targeted for the face are also indicated for treatment of TMD as a procedure combined with other therapies (e.g., electrotherapy, physiotherapy, temporomandibular joint mobilization, and facial massage) to improve pain sensitivity [11-13].

Phototherapy, such as low-level laser therapy (LLLT) and light-emitting diode (LED) therapy, are recently developed options to treat FM and TMD [14]. LLLT is assumed to modulate several signaling pathways and physiologic mechanisms involved in analgesia [15-17]. This therapeutic approach seems to increase $\beta$-endorphin levels, lymphatic flow, and blood supply. Moreover, LLLT is reported to reduce bradykinin, histamine release, swelling, pain-associated molecules, and inflammation phase and to induce muscle relaxation $[18,19]$. Similar results have been shown with LED therapy, which has a minor cost and better equipment durability [20].

A comprehensive review of the literature revealed no studies evaluating the role of phototherapy with multiple light sources (LLLT and LED) on the same device in the pain condition in patients with FM and TMD. In addition, there are no prior data involving a combined intervention of phototherapy and exercise training in patients with FM and TMD. The hypothesis of the proposed study is that combined intervention is capable of improving chronic pain in patients with FM and TMD. Knowledge regarding the clinical effects of these interventions can contribute to improved rehabilitation and quality of life among such patients.

\section{Objective}

In this trial, we seek to assess the potential role of exercise training plus phototherapy to reduce chronic pain in women with FM and TMD.

\section{Methods/Design \\ Study design}

The EXTRAPHOTO trial (ClinicalTrials.gov identifier NCT02279225) is a multicenter randomized controlled clinical trial comparing the use of exercise training, phototherapy, and exercise training plus phototherapy for pain control in women with two comorbidities (FM and TMD). As part of a simultaneous recruitment process, in the EXTRAPHOTO trial we will identify participants with target disorders in a database of the Unified Health System of Sao Paulo, Brazil. A preliminary analysis showed that patients at three centers with FM specialties are eligible: (1) the Rheumatology Clinic of Nove de Julho University, Sao Paulo, Brazil; (2) the basic health center of Sao Paulo, Brazil; and (3) the basic health center of Sao Paulo in Sao Bernardo do Campo, Brazil. The study procedure will follow the guidelines of the Consolidated Standards of Reporting Trials statement. All participants will receive data on the study aims and experimental procedures. We will obtain signed, written informed consent forms from each patient before inclusion in the study. The trial scheme is detailed in Fig. 1.

\section{Population and sample size}

In a literature review, we found that FM prevalence is higher in women between ages 35 and 60 years than in men [21]. All women patients aged $\geq 35$ years and referred for FM and TMD diagnostic evaluation were eligible for inclusion in the present trial. Patient eligibility comprises an evaluation of medical history, physical 


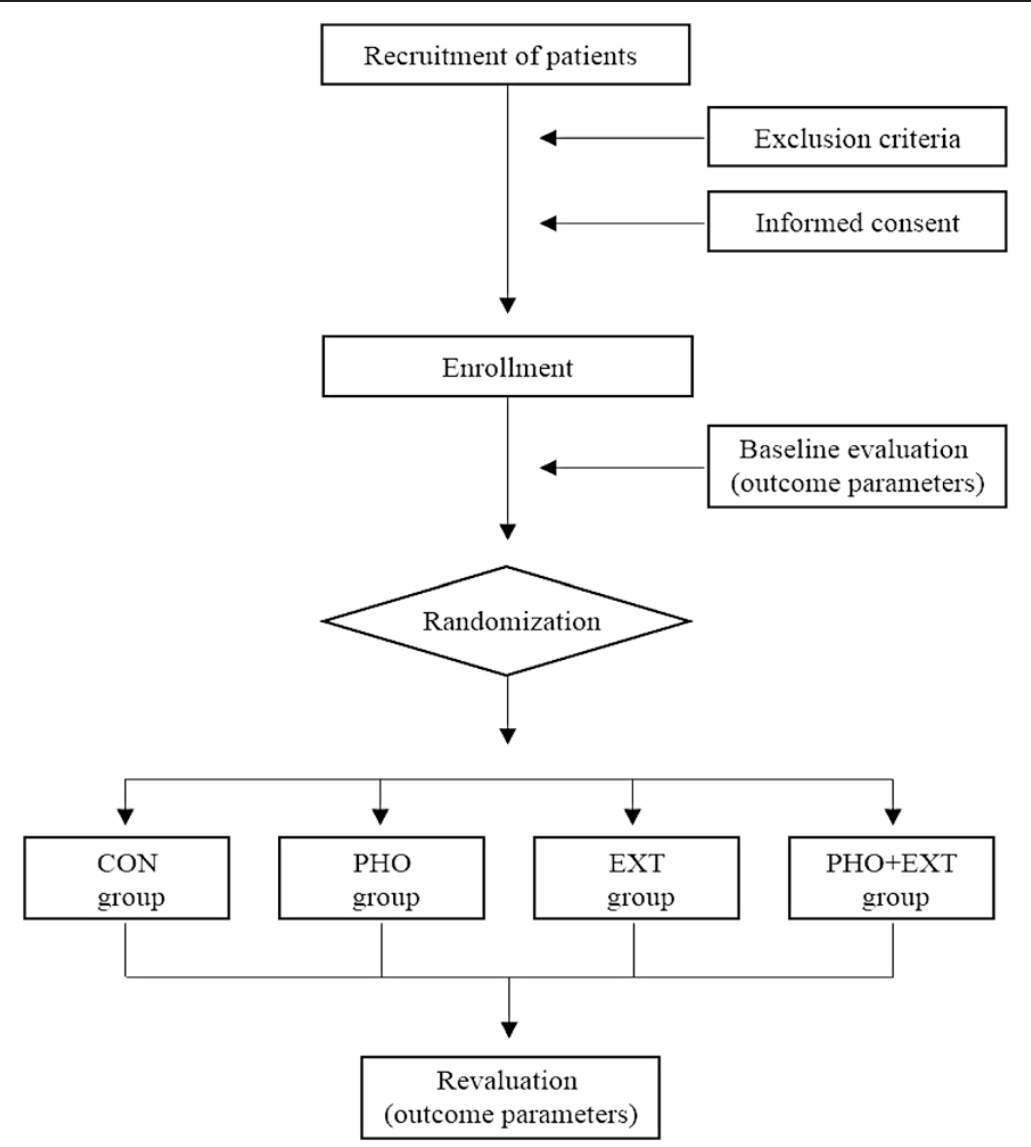

Fig. 1 Trial scheme. CON, Control; EXT, Exercise training; PHO + EXT, Phototherapy plus exercise training; PHO, Phototherapy

examination, and dental and rheumatologic routine evaluations. Moreover, patients had to have undergone FM screening as reported by the American College of Rheumatology using the Fibromyalgia Impact Questionnaire (FIQ) 1. The TMD diagnosis had to be reported on the basis of TMD research diagnostic criteria (axes I and II) [22]. The sample size calculation was based on a previous study by Consalter et al. [23] in which the TMD prevalence was $80 \%$ in patients with FM. A $95 \%$ reliability and a $10 \%$ error rate was assumed to estimate the sample size (n) as follows [24]:

$$
n=\left(\frac{z_{a / 2} \sqrt{p(1-p)}}{\varepsilon}\right)^{2}=\left(\frac{1.96 * \sqrt{0.8 * 0.2}}{0.1}\right)^{2}
$$

A total of 60 women will be randomized for this trial, with 20 women allocated per group.

\section{Recruitment and inclusion criteria}

The recruitment methods include direct referral of patients who consult for FM, in which TMD also will be investigated. A rheumatologist and a dentist will make the diagnoses of FM and TMD, respectively. A physician at each FM medical center will participate. A compatible diagnosis of FM will be obtained on the basis of evaluation criteria similar to those previously well defined [25]. The recruitment of 60 patients is planned to be finished within 24 months. Inclusion criteria are as follows: women $\geq 35$ years of age presenting with at least a 5-year diagnosis of FM and TMD, optimized drug treatment, cognitive independence to respond to inquiries, functionally independent regarding daily physical activity, availability and ability to fully comply with the training process and phototherapy, and no contraindication to the research procedures. All centers will be informed about the EXTRAPHOTO trial and its inclusion criteria during department meetings. Exclusion criteria are patients in a prior regular and structured physical activity program; missing more than three times from treatment; presence of psychiatric disorders, missing teeth, and/or use of dentures; a history of trauma to the face; currently undergoing orthodontic intervention; and any contraindication to exercise or phototherapy. The FM symptoms can be confused with other disorders. Thus, women for whom there are suspicions of any of the following disorders will be excluded: osteoarthritis, 
bursitis, tendinitis, rheumatoid arthritis, palindromic rheumatism, polymyalgic rheumatic disease, hydroxyapatite crystal diseases, systemic lupus erythematous, dermatomyositis-polymyositis complex, Lyme disease, hypothyroidism or hyperthyroidism, hyperparathyroidism, previous history of hepatitis, and history for Epstein-Barr virus infection. Sjögren, McArdle, Addison, Cushing, and paraneoplastic syndromes will also be exclusion criteria.

\section{Randomization, blinding, and experimental groups}

The EXTRAPHOTO trialists will enroll patients immediately after diagnosis of the two comorbidities and referral from recruitment centers. Patient enrollment and randomization, as well as data management, will be carried out by the Program in Biophotonics Applied to Health Sciences of the Nove de Julho University. The blinding will be applied to patients receiving phototherapy and a researcher reported to guide the exercise training. Thus, a researcher will be responsible for programming the phototherapy device, which can be turned on (phototherapy) or off (placebo) prior to application based on the randomization. A second researcher will guide the exercise training and will be blinded for phototherapy and/or placebo procedures. A third investigator will be blinded to allocation and will independently assess the outcomes. The statistical analysis will be performed by a fourth researcher, who will also be blinded to allocation of the patients.

The eligible participants will be instructed not to change their lifestyle or pharmacologic therapy during the study and will be randomized to one of the following groups:

1. Control $(\mathrm{CON})$ : patients not subjected to any intervention; the phototherapy device will be turned off (placebo) as a blinded procedure for these participants

2. Phototherapy (PHO): patients subjected to phototherapy

3. Exercise training (EXT): patients subjected to aerobic exercise; the phototherapy device will be turned off (placebo) as a blinded procedure for these participants

4. Phototherapy plus exercise training $(\mathrm{PHO}+\mathrm{EXT})$ : patients subjected to phototherapy and aerobic exercise training

\section{Interventions}

The trial will be 10 weeks long, during which time patients will undergo two sets of phototherapy, exercise, or placebo procedures per week. Phototherapy will be applied 30 min before each exercise bout, and treatment sessions will be carried out Tuesdays and Thursdays each week. For eligible patients, the research will record outcome parameters at baseline prior randomization and $48 \mathrm{~h}$ after the last day of intervention. This evaluator will be blinded to the allocation of the patients into the respective groups.

\section{Assessment and result reliability}

Two therapists will guide the interventions at each basic health center. To ensure the feasibility of the study and the reliability of the results, all therapists and researchers will be trained in data collection procedures before the start of the trial. Moreover, the results will be analyzed by an independent investigator without knowledge of the allocation of patients to their experimental groups.

\section{Phototherapy}

Phototherapy will be carried out with a PainAway ${ }^{\text {Tm }}$ ninediode cluster portable device (Multi Radiance Medical, Solon, OH, USA). This portable device was specifically designed for pain relief and has two operating modes: mild and severe. Because of the characteristics of pain that patients with FM, we decided to use the device at the mild setting. The device has two identical application hand pieces-one active tip and the other for placebo procedure without energy, both equipped with a similar sound device. These equipment pieces are required to blinding applicator research and patients. Thus, the researcher who will apply phototherapy and the patient will not know which tip was used (active or placebo). The irradiation will be applied only in the tender points in which pain has been reported by the participants. These tender points can be occipital, cervical (near the C7), trapezius, supraspinatus, second costochondral joint, lateral epicondyle and gluteal/sacrum, and greater trochanter on the medial knee border. The temporomandibular joint (bilaterally) will be another irradiation target because of TMD. Each point will be irradiated for $300 \mathrm{~s}$, in which $39.3 \mathrm{~J}$ of total energy will be delivered for each irradiation point. An independent researcher will be responsible for controlling the on or off phototherapy device because therapists and patients will be blinded to the procedure. The applicator researcher and patient will be wearing eye protection devices. The CON group will be subjected to the same procedures as the groups subjected to the PHO intervention. The phototherapy device to be used is shown in Fig. 2. All parameters of the phototherapy device are shown in the Table 1.

\section{Exercise training protocol}

The EXT protocol will be carried out according to the guidelines of the American College of Sports Medicine [26], and a previously published protocol will be applied 


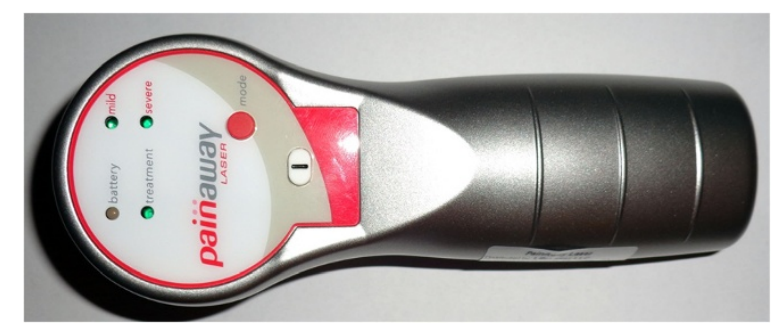

Fig. 2 Phototherapy device that will be used to treat pain

for the treatment of tender points of the patients with FM [25]. The protocol consists of stretching and aerobic exercise two times per week for 10 weeks. Active static stretching will be applied to the following muscle groups: biceps, trapezius, latissimus dorsi, pectoralis,

Table 1 Phototherapy parameters

\begin{tabular}{|c|c|}
\hline Parameters & Pulse \\
\hline & 1 Superpulsed infrared \\
\hline Wavelength of lasers (nm) & 905 \\
\hline Frequency $(\mathrm{Hz})$ & 1000 \\
\hline Average optical output (mW) & 0.9 \\
\hline Power density $\left(\mathrm{mW} / \mathrm{cm}^{2}\right)$ & 2.25 \\
\hline Peak power $(W)$ & 8.5 \\
\hline Dose (J) & 0.3 \\
\hline Energy density $\left(\mathrm{J} / \mathrm{cm}^{2}\right)$ & 0.75 \\
\hline Spot size of laser $\left(\mathrm{cm}^{2}\right)$ & 0.4 \\
\hline Number of LEDs & 4 Red \\
\hline Wavelength of LEDs (nm) & $640( \pm 10)$ \\
\hline Frequency $(\mathrm{Hz})$ & 2 \\
\hline Average optical output (mW), each & 15 \\
\hline Power density $\left(\mathrm{mW} / \mathrm{cm}^{2}\right)$, each & 16.66 \\
\hline Dose $(J)$, each & 4.5 \\
\hline Energy density $\left(\mathrm{J} / \mathrm{cm}^{2}\right)$, each & 5 \\
\hline Spot size of LED $\left(\mathrm{cm}^{2}\right)$, each & 0.9 \\
\hline Number of LEDs & 4 Infrared \\
\hline Wavelength of LEDs (nm) & $875( \pm 10)$ \\
\hline Frequency $(\mathrm{Hz})$ & 16 \\
\hline Average optical output (mW), each & 17.5 \\
\hline Power density $\left(\mathrm{mW} / \mathrm{cm}^{2}\right)$, each & 19.44 \\
\hline Dose $(J)$, each & 5.25 \\
\hline Energy density $\left(\mathrm{J} / \mathrm{cm}^{2}\right)$, each & 5.83 \\
\hline Spot size of LED $\left(\mathrm{cm}^{2}\right)$, each & 0.9 \\
\hline Magnetic field (mT) & 35 \\
\hline Treatment time (s) & 300 \\
\hline Aperture of device $\left(\mathrm{cm}^{2}\right)$ & 4 \\
\hline Total energy delivered $(J)$ & 39.3 \\
\hline
\end{tabular}

LED light-emitting diode paraspinal, hamstrings, and quadriceps. Each stretching exercise will be performed three times for $30 \mathrm{~s}$, and $30 \mathrm{~s}$ of rest will be allowed between each stretch. The stretch will be conducted to produce mild discomfort. The aerobic exercise will be carried out on an electronic motorized drive (LX-150; Movement, Sao Paulo, Brazil) without inclination for $30 \mathrm{~min}$ per session. The exercise will be guided at an intensity of $75 \%$ of age-predicted maximum heart rate (220 - age in years).

Exercises for TMD will be carried out as reported in detail elsewhere [27]. Exercises will be performed with the patient in a sitting position and repeated three times for every movement. A maximum oral opening will be required for the first exercise; the second exercise will be a tongue slippage on the palate; and the third exercise will be oral lateralization to the right and left with contraction of the masseter muscle. This exercise will be conducted with the participant's mouth filled with air for $3 \mathrm{~s}$. Ultimately, circular fingertip motions will be applied with slight pressure on the temporomandibular joint and masseter muscle [27, 28].

\section{Outcome parameters}

The outcome parameters described below will be evaluated at baseline and $24 \mathrm{~h}$ after the last day of the experimental protocol.

\section{Personal and anthropometric data}

We will collect data on participants' occupation, education level, marital status, social security number, home address, age, body weight, and height.

\section{Overall parameters of pain}

To assess overall parameters of pain, we will gather information on daily duration of pain, pain tender points, number of days with a high pain level, sleep quality, main features that show accentuation or worsening of pain, incidence of stiffness and pain, jaw locking, and morning fatigue.

\section{Visual analogue scale}

We will use a visual analogue scale (VAS) to assess pain level. The VAS consists of a limited strip of $10 \mathrm{~cm}$ in length. This strip has at its ends the anchor terms no pain and worst pain possible. The patient will be instructed to report the level of pain sensation to a point along this line, with the scores ranging from 0 to 10 and obtained by measuring the distance between the end anchored by the term without pain and the point marked by the participant [29]. Additional analysis will include changes from randomization to the end of withdrawal (week 10) in an adapted Portuguese version of the McGill Pain Questionnaire (McPQ) [30]. 


\section{Algometry}

A digital algometer (DD-200; Instrutherm, Sao Paulo, Brazil) will be used to evaluate pain sensibility through the application of pressure. The algometer will be positioned to read specific FM tender points and TMD joints using the rubber tip measuring $1 \mathrm{~cm}^{2}$ in direct contact with the skin. A gradually increasing pressure will be applied to all points until the patient reports feeling pain, at which point the researcher will stop applying pressure and display values will be recorded. The procedures will be performed only once to each point, and a 30-s rest period will be given between readings [29].

\section{Fibromyalgia symptoms and quality of life}

Outcome parameters will be evaluated with the FIQ. The FIQ is a multidimensional instrument developed to assess several FM symptoms (pain, fatigue, stiffness, tenderness, sleep disorders, depressed mood, anxiousness, problems with memory, imbalance, sensitivity to nonpainful stimuli, and performing daily tasks) [29]. The Medical Outcomes Study 36-item Short Form Health Survey (SF-36) will also be used [29].

\section{Salivary serotonin}

Concentration of serotonin in the saliva is relevant to nociception and the pathogenesis of chronic pain syndromes seen in FM [31]. Thus, mean serotonin changes from randomization to the end of withdrawal will be evaluated in $25-\mu \mathrm{l}$ salivary samples by using a competitive enzyme-linked immunosorbent assay (ELISA) according to the manufacturer's instructions (Human Serotonin ST ELISA Kit; MyBioSource, San Diego, CA, USA). The samples will be collected with cotton swabs into plastic tubes without any stimulation and frozen $\left(-80{ }^{\circ} \mathrm{C}\right)$. Patients will be instructed to rinse their mouths with water and not to eat or drink 30 min before the samples are collected. The ELISA plates will be read with a SpectraMax Plus 384 spectrophotometer (Molecular Dynamics, Sunnyvale, CA, USA) at a wavelength of $400 \mathrm{~nm}$ [31].

\section{Statistical analysis}

Pain will be the primary endpoint and will be determined using a VAS, an algometer, the FIQ, and the McPQ. Quality of life as assessed by SF-36, salivary serotonin level, and overall parameters of pain will be secondary endpoints. Statistical analysis will be performed using IBM SPSS software (version 13.0; IBM, Armonk, NY, USA), and a two-sided $P$ value $<0.05$ will be considered significant. The intragroup and intergroup comparisons will be carried out with two-way repeatedmeasures analysis of variance with post hoc Bonferroni correction or the Kruskal-Wallis test with a post hoc Dunn test. The choice of tests will be based on the distribution (normal or non-normal) of the data by the Shapiro-Wilk test. For categorical data, $\chi^{2}$ statistics will be used.

\section{Ethical approval}

The ethics committee of the Nove de Julho University (Sao Paulo, Brazil) reviewed and approved this study protocol (protocol number 419.828/2013). The trial was conceived and will be conducted according to the principles set forth in the Declaration of Helsinki [32]. All patients will be requested to provide written informed consent prior to randomization, using standard forms. Patients may withdraw from the study at any time without penalty. Our standpoint is that any intervention has beneficial effect. It is an ethical issue in which the control group knows the positive results of the study. Therefore, the control group will be given the opportunity to receive intervention after the end of the trial. The trial is registered at ClinicalTrials.gov under the number NCT02279225 (27 October 2014).

\section{Discussion}

The findings this trial are predicted to offer evidence regarding the role of phototherapy and exercise training as well as a combined intervention in a multimodal management program for patients with FM and TMD. This is a target population because TMD prevalence is higher in patients with FM [28]. Pain is a the main symptom of these conditions [33-35], and it limits the patient's activities of daily living, such as walking, carrying objects, occupational, eating, talking, yawning, and smiling, as well as a worsened in quality of life $[35,36]$. Moreover, pain is associated to sleep disturbances, fatigue, and mood and memory disorders [36, 37]. Hence, we consider that pain may represent the main endpoint in the EXTRAPHOTO trial.

It has been proposed that chronic pain disorders are driven mainly by alterations in the central nervous system in which serotonin is considered the most important neurotransmitter that modulates endogenous mechanisms [38]. Because several studies have shown that serotoninergic dysfunction can mediate the pathophysiology of FM and TMD [38, 39], salivary serotonin level will represent a secondary endpoint in the EXTRAPHOTO trial. To the best of our knowledge, no previous randomized controlled trial has evaluated whether phototherapy and/or exercise training can modulate endogenous serotonin in patients with FM and TMD [40].

The EXTRAPHOTO trial is the first randomized controlled study evaluating the role of phototherapy, exercise training, and a combination of these interventions for chronic pain in patients with FM and TMD. In fact, the results of this trial will provide valuable clinical evidence for objective assessment of the potential benefits and risks of procedures. The results will be published after the trial is completed. 


\section{Trial status}

The proposed study is presently under development, and individuals are being allocated in the experimental groups.

\author{
Abbreviations \\ CON: Control; ELISA: Enzyme-linked immunosorbent assay; EXT: Exercise \\ training; FIQ: Fibromyalgia impact questionnaire; FM: Fibromyalgia; \\ LED: Light-emitting diode; LLLT: Low-level laser therapy; PHO: Phototherapy; \\ PHO + EXT: Phototherapy plus exercise training; TMD: Temporomandibular \\ dysfunction; VAS: Visual analogue scale.
}

\section{Competing interests}

ECPL-J receives research support from Multi Radiance Medical (Solon, $\mathrm{OH}$, USA), a laser device manufacturer. The remaining authors declare that they have no competing interests.

\section{Authors' contributions}

MMS: study conception and design, data collection and analysis, and manuscript writing. RA: study conception and design, financial support, and manuscript writing. ECPL-J: study conception and design, financial support, and manuscript writing. PTCC: data collection and analysis and critical revision of the manuscript. JAS: data collection and analysis and critical revision of the manuscript. SKB: data collection and analysis and critical revision of the manuscript. LVFO: study conception and design, financial support, and manuscript writing. CASC: study conception and design, financial support, and manuscript writing. ELAA: data collection and analysis and critical revision of the manuscript. DSB: study conception and design, financial support, and manuscript writing. AJS: study conception and design, financial support, and manuscript writing. All authors read and approved the final manuscript.

\section{Acknowledgments}

We are grateful to all individuals involved in the study, the researchers, volunteers and Nove de Julho University (UNINOVE). We also thank the Coordination for the Improvement of Higher Education Personnel (CAPES) Foundation for the student grant. All authors read and approved the final manuscript.

\section{Received: 4 February 2015 Accepted: 19 May 2015 Published online: 04 June 2015}

\section{References}

1. Wolfe F, Smythe HA, Yunus MB, Bennett RM, Bombardier C, Goldenberg DL, et al. The American College of Rheumatology 1990 criteria for the classification of fibromyalgia. Arthritis Rheum. 1990;33(2):160-72.

2. Cassisi G, Ceccherelli F, Atzeni F, Sarzi-Puttini P. Complementary and alternative medicine in fibromyalgia: a practical clinical debate of agreements and contrasts. Clin Exp Rheumatol. 2013;31(6 Suppl 79):S134-52.

3. Arnold L, Stanford S, Welge J, Crofford L. Development and testing of the fibromyalgia diagnostic screen for primary care [abstract]. J Pain. 2011;12(4 Suppl):8. doi:10.1016/j.jpain.2011.02.031 .

4. Clauw DJ. Fibromyalgia. JAMA. 2014;311:1547-55.

5. Fraga BP, Santos EB, Farias Neto JP, Macieira JC, Quintans Jr LJ, Onofre AS, et al. Signs and symptoms of temporomandibular dysfunction in fibromyalgic patients. J Cranio Fac Surg. 2012;23:615-8.

6. Gür A. Physical therapy modalities in management of fibromyalgia. Curr Pharm Des. 2006;12:29-35.

7. Eisenlohr-Moul TA, Crofford LJ, Howard TW, Yepes JF, Carlson CR, de Leeuw R. Parasympathetic reactivity in fibromyalgia and temporomandibular disorder: associations with sleep problems, symptom severity, and functional impairment. J Pain. 2015;16(3):247-57.

8. Batista JS, Borges AM, Wibelinger AM. Tratamento fisioterapêutico na síndrome da dor miofascial e da fibromialgia. Rev Dor. 2012;13(2):170. doi:10.1590/S1806-00132012000200014. Portuguese.

9. Orlandi AC, Ventura C, Gallinaro AL, Costa RA, Lage LV. Improvement in pain, fatigue, and subjective sleep quality through sleep hygiene tips in patients with fibromyalgia. Rev Bras Reumatol. 2012;52(5):672-8.
10. Busch AJ, Barber KAR, Overend TJ, Peloso PMJ, Schachter CL. Exercise for treating fibromyalgia syndrome. Cochrane Database Syst Rev. 2007:4:CD003786.

11. Häuser W, Klose P, Langhorst J, Moradi B, Steinbach M, Schiltenwolf M, et al. Efficacy of different types of aerobic exercise in fibromyalgia syndrome: a systematic review and meta-analysis of randomised controlled trials. Arthritis Res Ther. 2010;12:R79.

12. Beckerman H, Bouter LM, van der Heijden GJ, de Bie RA, Koes BW. Efficacy of physiotherapy for musculoskeletal disorders: what can we learn from research? Br J Gen Pract. 1993;43:73-7.

13. De Boever JA, Nilner M, Orthlieb JD, Steenks MH. Educational Committee of the European Academy of Craniomandibular Disorders. Recommendations by the EACD for examination, diagnosis, and management of patients with temporomandibular disorders and orofacial pain by the general dental practitioner. J Orofac Pain. 2008;22:268-78.

14. John MT, Dworkin SF, Mand LA. Reliability of clinical temporomandibular disorder diagnoses. Pain. 2005;118:61-9.

15. Shinozaki EB, Paiva G, Zanin FAA, Brugnera Jr A. The electromyography evaluation in temporomandibular joint disease patients after laser therapy. RGO. 2006;54:334-9. Portuguese.

16. Camillo de Carvalho PT, Leal-Junior ECP, Alves ACA, De Melo Rambo CS, Sampaio LMM, Oliveira CS, et al. Effect of low-level laser therapy on pain, quality of life and sleep in patients with fibromyalgia: study protocol for a double-blinded randomized controlled trial. Trials. 2012;13:221.

17. Gür A, Karakoç M, Nas K, Cevik R, Saraç J, Demir E. Efficacy of low power laser therapy in fibromyalgia: a single-blind, placebo-controlled trial. Lasers Med Sci. 2002:17:57-61.

18. da Cunha LA, Firoozmand LM, da Silva AP, Camargo SE, Oliveira W. Efficacy of low-level laser therapy in the treatment of temporomandibular disorder Int Dent J. 2008:58:213-7.

19. Emshoff R, Bösch R, Pümpel E, Schöning H, Strobl H. Low-level laser therapy for treatment of temporomandibular joint pain: a double-blind and placebo-controlled trial. Oral Surg Oral Med Oral Pathol Oral Radiol Endod. 2008;105:452-6.

20. Herpich CM, Leal-Junior ECP, Amaral AP, de Paiva TJ, dos Santos Glória IP, Garcia MBS, et al. Effects of phototherapy on muscle activity and pain in individuals with temporomandibular disorder: a study protocol for a randomized controlled trial. Trials. 2014;15:491.

21. Cavalcante $A B$, Sauer JF, Chalot SD, Assumpção A, Lage LV, Matsutani LA et al. The prevalence of fibromyalgia: a literature review. Rev Bras Reumatol. 2006:46:40-8.

22. Dworkin SF, LeResche L. Research diagnostic criteria for temporomandibular disorders: review, criteria, examinations and specifications, critique. J Craniomandib Disor. 1992;6:301-55.

23. Consalter E, Sanches ML, Guimarães AS. Correlação entre disfunção temporomandibular e fibromialgia. Rev Dor. 2010;11(3):237-41. Portuguese

24. Levine DM, Berenson ML, Stephan D, editors. Estatística: teoria e aplicações usando Microsoft Excel em Português. Rio de Janeiro: LTC; 2000. p. 811. Portuguese.

25. Wolfe F, Clauw DJ, Fitzcharles MA, Goldenberg DL, Katz RS, Mease P, et al. The American College of Rheumatology preliminary diagnostic criteria for fibromyalgia and measurement of symptom severity. Arthritis Care Res. 2010;62:600-10.

26. Kraemer WJ, Adams K, Cafarelli E, Dudley GA, Dooly C, Feigenbaum MS, et al. American College of Sports Medicine: progression model in resistance training for healthy adults. Stand position. Med Sci Sports Exerc. 2002;34(2):364-80.

27. Biasotto-Gonzalez DA, Bérzin F. Electromyographic study of patients with masticatory muscles disorders, physiotherapeutic treatment (massage). Braz J Oral Sci. 2004;3:516-21.

28. Bae $Y$, Park $Y$. The effect of relaxation exercises for the masticator muscles on temporomandibular joint dysfunction (TMD). J Phys Ther Sci. 2013;25:583-6.

29. Alves AMB, Natour J, Assis MR, Feldman D. Assessment of different instruments used as outcome measures in patients with fibromyalgia. Rev Bras Reumatol. 2012:52:501-6.

30. Pimenta CAM, Teixeira MJ. McGill Pain Questionnaire: proposal for adaptation into Portuguese. Rev Bras Anestesiol. 1997;47:177-86. Portuguese.

31. Fischer HP, Eich W, Russell IJ. A possible role for saliva as a diagnostic fluid in patients with chronic pain. Semin Arthritis Rheum. 1998;27:348-59. 
32. World Medical Association. World Medical Association Declaration of Helsinki: ethical principles for medical research involving human subjects. JAMA. 2013;310:2191-4.

33. Holst H, Arendt-Nielsen L, Mosbech H, Elberling J. Increased capsaicin-induced secondary hyperalgesia in patients with multiple chemical sensitivity. Clin J Pain. 2011;27:156-62.

34. Calandre EP, Rico-Villademoros F. The role of antipsychotics in the management of fibromyalgia. CNS Drugs. 2012;26:135-53. A published erratum appears in. CNS Drugs. 2013;27(6):491.

35. Shedden Mora M, Weber D, Borkowski S, Rief W. Nocturnal masseter muscle activity is related to symptoms and somatization in temporomandibular disorders. J Psychosom Res. 2012;73:307-12.

36. Türp JC, Motschall E, Schindler HJ, Heydecke G. In patients with temporomandibular disorders, do particular interventions influence oral health-related quality of life? A qualitative systematic review of the literature. Clin Oral Implants Res. 2007;18 Suppl 3:127-37.

37. Sarzi-Puttini P, Atzeni F, Di Franco M, Buskila D, Alciati A, Giacomelli C, et al. Dysfunctional syndromes and fibromyalgia: a 2012 critical digest. Clin Exp Rheumatol. 2012;30(6 Suppl 74):143-51.

38. Green AR. Neuropharmacology of 5-hydroxytryptamine. Br J Pharmacol. 2006;147 Suppl 1:S145-52.

39. de Freitas LV, Lopes AC, Piatto VB, Maniglia JV. Association of temporomandibular dysfunction with the 102T-C polymorphism in the serotonin receptor gene in Brazilian patients. Arch Med Sci. 2013:9:1013-8.

40. Gerdle B, Ghafouri B, Ernberg M, Larsson B. Chronic musculoskeletal pain: review of mechanisms and biochemical biomarkers as assessed by the microdialysis technique. J Pain Res. 2014;7:313-26.

\section{Submit your next manuscript to BioMed Central and take full advantage of:}

- Convenient online submission

- Thorough peer review

- No space constraints or color figure charges

- Immediate publication on acceptance

- Inclusion in PubMed, CAS, Scopus and Google Scholar

- Research which is freely available for redistribution 